\title{
ON THE CATEGORICAL BEHAVIOUR OF PREORDERED GROUPS
}

\author{
MARIA MANUEL CLEMENTINO, NELSON MARTINS-FERREIRA, AND ANDREA MONTOLI
}

\begin{abstract}
We study the categorical properties of preordered groups. We first give a description of limits and colimits in this category, and study some classical exactness properties. Then we point out a strong analogy between the algebraic behaviour of preordered groups and monoids, and we apply two different recent approaches to relative categorical algebra to obtain some homological properties of preordered groups.
\end{abstract}

\section{INTRODUCTION}

The categorical properties of topological groups, and more generally of topological models of semi-abelian algebraic theories, have been studied in $[3,4]$. This study allowed to understand several algebraic and homological properties of these structures. Despite the similarities in the topological flavour of the categories Top of topological spaces and Ord of preordered sets, analogous techniques are not applicable to preordered groups. Indeed, an important difference between topological groups and preordered ones is that the latter are not internal models in Ord of the theory of groups, since the inverse map is not monotone, in general. Because of this, several properties fail for preordered groups. For example, the category OrdGrp is not protomodular in the sense of [5], which means that the (split) short five lemma does not hold. In fact, a thorough study of the structure of split extensions in OrdGrp shows a great variety of such structures: in some cases, it is possible to equip the domain of a split epimorphism with uncountably many preorder structures that give rise to split extensions of preordered groups, while in some others there is no compatible preorder.

The aim of this paper is to study the categorical properties of OrdGrp, following an approach which is necessarily different from the one of [3,4]. First of all, from the properties of the forgetful functors between OrdGrp and the categories Grp of groups and Ord, we obtain a description of limits and colimits in OrdGrp, and we observe that there are two stable factorization systems: (Epi, Regular Mono) and (Regular Epi, Mono). In particular, OrdGrp is a regular category. Actually we can say more: it is normal, in the sense of [13], and efficiently regular [7], although not Barr-exact [1].

Moreover, using the fact that the preorder relation is uniquely determined by the submonoid of positive elements (called the positive cone), we get another forgetful functor, into the category $\mathbf{M o n}_{\text {can }}$ of monoids with cancellation, which has a left adjoint. This allows to identify a strong similarity between the algebraic and homological behaviour of OrdGrp and of the category Mon of monoids. In particular, two different relativizations of the classical categorical-algebraic notions give interesting information when applied to OrdGrp. The first one is the objectwise approach introduced in [22]: the idea is to identify, in a category with weak algebraic properties, some "good" objects, which satisfy stronger properties. Such objects are called Mal'tsev and 
protomodular objects [22]. It was shown in [22] that the Mal'tsev objects and the protomodular objects in Mon are precisely the groups. Using this fact, we prove here that the Mal'tsev objects and the protomodular objects in OrdGrp are precisely those preordered groups whose submonoid of positive elements is a group or, in other terms, whose preorder relation is symmetric. If we restrict our attention to strictly ordered groups, the "good" objects are the discrete groups.

The second approach is the study of protomodularity with respect to a pullback stable class of points (i.e. split epimorphisms with a fixed section), following [10]. Again, the first example of this situation was observed in Mon, w.r.t. the class of Schreier points [21]. Here we identify two suitable classes of points, one obtained by imposing that the restriction to the positive cones is a Schreier point, the other by considering the internalization of the Schreier condition in the category Mon(Ord) of internal monoids in Ord. With respect to both classes, OrdGrp turns out to be an $S$-protomodular category (in the sense of [10]). This allows to extend the homological results obtained in $[10,11]$ to OrdGrp.

\section{On the CATEGORY OrdGrp OF PREORDERED GROUPS}

We denote by OrdGrp the category whose objects are preordered groups, namely (not necessarily abelian) groups $G$ equipped with a preorder relation (i.e. a reflexive and transitive relation $) \leq$ such that the group operation is monotone:

$$
\forall a, b, c, d \in G, \quad a \leq c, b \leq d \quad \Rightarrow \quad a+b \leq c+d,
$$

and whose arrows are monotone group homomorphisms.

It is well known that the preorder relation of a preordered group is completely determined by the positive cone of $G$, which is the subset $P_{G}$ of positive elements of $G$, that are the elements $a \in G$ such that $a \geq 0$ :

Proposition 2.1. For a group $G$, to give a preorder relation $\leq$ on $G$ such that $(G, \leq) \in$ OrdGrp is equivalent to giving a submonoid $P_{G}$ of $G$ which is closed under conjugation in $G$ : for all $a \in P_{G}$ and all $b \in G, b+a-b \in P_{G}$.

Proof. Suppose that $(G, \leq) \in \operatorname{OrdGrp}$ and define $P_{G}=\{a \in G \mid a \geq 0\}$. It is clear that $P_{G}$ is a submonoid of $G$ closed under conjugation.

Conversely, let $P$ be a submonoid of a group $G$ closed under conjugation. We define a relation $\leq$ on $G$ by putting:

$$
a \leq b \quad \Leftrightarrow \quad b-a \in P .
$$

This relation is clearly reflexive. For transitivity, suppose that $a \leq b$ and $b \leq c$. Then $b-a, c-b \in$ $P$. Hence $c-a=c-b+b-a \in P$ and so $a \leq c$. Moreover, the group operation is monotone. Indeed, if $a \leq c$ and $b \leq d$, then $c-a, d-b \in P$. Hence $c+b-(a+b)=c-a \in P$, so that $a+b \leq c+b$. Furthermore

$$
c+d-(c+b)=c+d-b-c \in P,
$$

because $d-b \in P$ and $P$ is closed under conjugation. Hence $c+b \leq c+d$. This concludes the proof.

We will denote preordered groups by $X, Y$, etc., using $P_{X}, P_{Y}$, etc. for the corresponding positive cones. It is immediate to see that a morphism $f: X \rightarrow Y$ in OrdGrp restricts to a 
monoid homomorphism $P_{X} \rightarrow P_{Y}$. Conversely, given groups $G$ and $H$ with submonoids $M$ and $N$ closed under conjugation, a group homomorphism $f: G \rightarrow H$ whose restriction to $M$ takes values in $N$ induces a morphism in OrdGrp between $G$ and $H$ with the preorders determined by $M$ and $N$, respectively. Hence, the category OrdGrp is isomorphic to the category whose objects are pairs $(G, M)$, where $G$ is a group and $M$ is a submonoid of $G$ closed under conjugation, and whose morphisms $(G, M) \rightarrow(H, N)$ are group homomorphisms $f: G \rightarrow H$ such that $f(M) \subseteq N$.

Remark 2.2. If a group $G$ has only elements of finite order (in particular, if $G$ is finite), then every preorder $\leq$ which makes $G$ a preordered group is symmetric, hence an equivalence relation. Indeed, given $a \neq 0$, if $a \geq 0$ then, by monotonicity of the group operation, every element of the form $n a$, with $n \in \mathbb{N}$, is positive. Since $a$ has finite order, its inverse is one of these elements, hence $-a$ is positive too. This means that the submonoid $P_{G}$ is a group, which is equivalent to saying that the relation $\leq$ is symmetric.

Proposition 2.3. Consider the forgetful functors $\mathrm{U}_{1}$ : OrdGrp $\rightarrow$ Grp and $\mathrm{U}_{2}$ : OrdGrp $\rightarrow$ Ord, with $\mathrm{U}_{1}$ forgetting the preorder and $\mathrm{U}_{2}$ the group structure. The functor $\mathrm{U}_{1}$ is topological while $\mathrm{U}_{2}$ is monadic. We have therefore the following commutative diagram

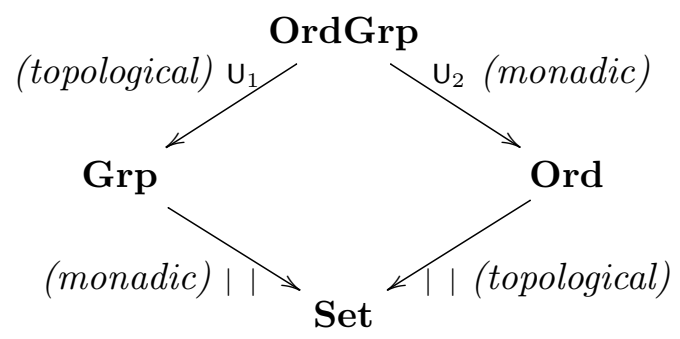

Proof. To show that $\mathrm{U}_{1}$ is a topological functor, let $\left(f_{i}: G \rightarrow X_{i}\right)_{i \in I}$ be a family of group homomorphisms, with $X_{i}, i \in I$, preordered groups. Then $P_{G}=\left\{y \in G \mid f_{i}(y) \in P_{X_{i}}\right.$ for every $\left.i \in I\right\}$ is a submonoid of $G$ closed under conjugation, and this defines clearly the $\mathrm{U}_{1}$-initial lifting for $\left(f_{i}\right)$. Topologicity of $\mathbf{U}_{1}$ gives that, with Grp, also OrdGrp is complete and cocomplete.

To show that $\mathrm{U}_{2}$ is monadic, we will use [14, Theorem 2.4]. (a) $\mathrm{U}_{2}$ has a left adjoint $\mathrm{L}_{2}$ : Ord $\rightarrow$ OrdGrp: it sends a preorder $A$ to the free group $\mathrm{F}(A)$ on the set $A$, equipped with the preorder determined by the submonoid of $\mathrm{F}(A)$ obtained by closing under addition and conjugation the set of the elements of the form $b-a$ for all $a, b \in A$ such that $a \leq b$.

(b) $\mathrm{U}_{2}$ reflects isomorphisms: given a morphism $f: X \rightarrow Y$ in OrdGrp, if $\mathrm{U}_{2}(f)$ is an isomorphism in Ord then $f$ is a bijective homomorphism and, for every $x, x^{\prime} \in X, x \leq x^{\prime}$ if and only if $f(x) \leq f\left(x^{\prime}\right)$. Therefore its inverse map is a monotone homomorphism, and so $f$ is an isomorphism in OrdGrp.

(c) OrdGrp has and $\mathrm{U}_{2}$ preserves coequalizers of all $\mathrm{U}_{2}$-contractible coequalizer pairs. First recall that the functor $\mid$ : Grp $\rightarrow$ Set is monadic. Given morphisms $f, g: X \rightarrow Y$ in OrdGrp such that $\mathrm{U}_{2}(f), \mathrm{U}_{2}(g)$ is a contractible pair in Ord, we know that the coequalizer $q: Y \rightarrow Q$ in OrdGrp is preserved by $U_{1}$, and so it is also preserved by $\mid \cdot U_{1}$, since || is monadic and $\left|\mathbf{U}_{1}(f)\right|,\left|\mathbf{U}_{1}(g)\right|$ are a contractible pair in Set. Therefore $\mathbf{U}_{2}(q)$, as a split epimorphism that coequalizes $\left|\mathbf{U}_{1}(f)\right|,\left|\mathbf{U}_{1}(g)\right|$ in Set, is the coequalizer of $\mathbf{U}_{2}(f), \mathbf{U}_{2}(g)$ in Ord.

We collect below properties of OrdGrp that follow from this proposition.

Remark 2.4. (1) The functor $\mathrm{U}_{1}$ : OrdGrp $\rightarrow$ Grp has both a left and a right adjoint. The left adjoint $\mathrm{L}_{1}: \operatorname{Grp} \rightarrow$ OrdGrp equips a group $G$ with the discrete order: $a \leq b$ if and 
only if $a=b$. The right adjoint $\mathrm{R}_{1}: \mathbf{G r p} \rightarrow$ OrdGrp equips a group $G$ with the total relation: $a \leq b$ for all $a, b \in G$. It is immediate to see that both relations are compatible with the group operation. In the first case, $P_{G}=\{0\}$, while in the second one $P_{G}=G$.

(2) OrdGrp is complete and cocomplete, as stated in the proof of Proposition 2.3.

(3) Limits are preserved by both forgetful functors. Therefore the product $X \times Y$, of two ordered groups $X$ and $Y$, is the direct product of groups equipped with the relation $\leq$ given by:

$$
\left(x_{1}, y_{1}\right) \leq\left(x_{2}, y_{2}\right) \quad \Leftrightarrow \quad x_{1} \leq x_{2} \text { and } y_{1} \leq y_{2}
$$

that is, $P_{X \times Y}=P_{X} \times P_{Y}$. Infinite products are obtained similarly. The equalizer of a pair $f, g: X \rightarrow Y$ of parallel morphisms in OrdGrp is the equalizer in Grp equipped with the preorder induced by the one of $X$.

(4) Colimits are preserved by $\mathrm{U}_{1}$ : OrdGrp $\rightarrow \operatorname{Grp}\left(\right.$ but not by $\mathrm{U}_{2}$ ), so they are formed like in Grp and equipped with the suitable preorder, as outlined next.

Coequalizers are easily described. Given a pair of morphisms $f, g: X \rightarrow Y$, let $q: \mathrm{U}_{1}(Y) \rightarrow Q$ be the coequalizer in Grp of $\mathrm{U}_{1}(f), \mathrm{U}_{1}(g)$. Putting $P_{Q}=q\left(P_{Y}\right)$, it is easy to check that $P_{Q}$ is a submonoid of $Q$ closed under conjugation, hence it makes $\left(Q, P_{Q}\right)$ a preordered group; and $q: Y \rightarrow\left(Q, P_{Q}\right)$ is clearly a morphism in OrdGrp. The universal property is easily checked.

Coproducts are a bit more difficult. Given two preordered groups $X$ and $Y$, its coproduct is the free product of $\mathrm{U}_{1}(X)$ and $\mathrm{U}_{1}(Y)$ in Grp equipped with the positive cone obtained as the closure, under (internal) addition and conjugation, of the disjoint union of $P_{X}$ and $P_{Y}$; it can be, of course, also described as the intersection of all the submonoids containing both $P_{X}$ and $P_{Y}$ and closed under conjugation. Infinite coproducts are obtained similarly.

(5) In OrdGrp a morphism $f: X \rightarrow Y$ is an epimorphism if and only if it is surjective: the preservation of colimits by $U_{1}$ and its faithfulness imply that $U_{1}$ preserves and reflects epimorphisms; therefore $f$ is an epimorphism in OrdGrp if and only if $U_{1}(f)$ is an epimorphism in Grp, that is, $f$ is surjective. Regular monomorphisms in OrdGrp are the morphisms $f: X \rightarrow Y$ that are injective with $P_{X}=f^{-1}\left(P_{Y}\right)$. It is easily seen that (Epi, Reg Mono) is a stable factorization system in OrdGrp: every $f: X \rightarrow Y$ can be factored as

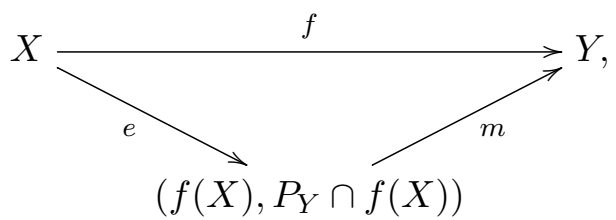

and epimorphisms are pullback stable (just because surjective homomorphisms are pullback stable in Grp).

(6) As shown above, a morphism $f: X \rightarrow Y$ is a regular epimorphism if and only if it is surjective and $f\left(P_{X}\right)=P_{Y}$; and $f$ is a monomorphism exactly when it is an injective map. It is easy to check that (Reg Epi, Mono) is a stable factorization system in OrdGrp. Indeed, every $f: X \rightarrow Y$ can be factored as

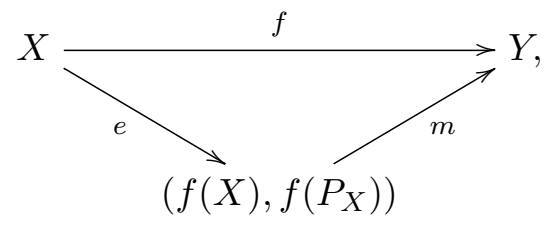


and regular epimorphisms are pullback-stable: consider the following pullback

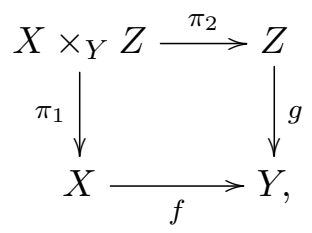

where $f$ is a regular epimorphism. If $z \in P_{Z}$, then $g(z) \in P_{Y}$ and so there exists $x \in P_{X}$ with $f(x)=g(z)$.

Proposition 2.5. The category OrdGrp is a regular category. Moreover, it is normal.

Proof. As stated above, OrdGrp is a (finitely) complete category with a stable factorization system (Reg Epi, Mono), hence it is regular in the sense of Barr [1]. To show that it is normal in the sense of Z. Janelidze [13], we observe that it is pointed and that, for every regular epimorphism $f: X \rightarrow Y, \mathrm{U}_{1}(f)$ is a regular epimorphism in Grp, hence a normal epimorphism in Grp, and then, thanks to Remark 2.4.(4), $f$ is a normal epimorphism in OrdGrp.

Remark 2.6. The category OrdGrp is not Barr-exact [1]. Indeed, consider the following equivalence relation:

$$
\mathbb{Z} \times \mathbb{Z} \underset{p_{2}}{\stackrel{p_{1}}{\longleftarrow}\langle 1,1\rangle \longrightarrow} \mathbb{Z},
$$

where $\mathbb{Z}$ is equipped with the usual order and the positive cone of $\mathbb{Z} \times \mathbb{Z}$ is

$$
P=\{(x, x) \mid x \geq 0\} .
$$

This is an equivalence relation in OrdGrp which is not effective, because the morphism $\left\langle p_{1}, p_{2}\right\rangle$, which is the identity map as sets, is not an extremal monomorphism. However, OrdGrp is efficiently regular (see [7], where the name used was effectively regular), namely if $R$ is an effective equivalence relation over an object $X$ and $T$ is another equivalence relation over $X$ which is a regular subobject $j: T \longmapsto R$ of $R$ (i.e. $j$ is a regular monomorphism in OrdGrp), then $T$ is itself effective. Indeed, $T$ is a kernel pair of a morphism in Grp, since Grp is Barr-exact. Moreover, being $j$ a regular monomorphism in OrdGrp, $P_{T}=T \cap P_{R}$. The equivalence relation $R$ is effective in OrdGrp, hence $P_{R}=R \cap P_{X \times X}$, and so

$$
P_{T}=T \cap R \cap P_{X \times X}=T \cap P_{X \times X},
$$

which proves that $T$ is effective in OrdGrp.

Remark 2.7. From Remark 2.4 (6) it follows that a morphism $f: X \rightarrow Y$ in OrdGrp is an extremal epimorphism (which is the same as a strong - or a regular - epimorphism) if and only if it is an extremal epimorphism in Grp and its restriction to the positive cones is an extremal epimorphism in Mon. However, the same is not true for pairs, or families, of morphisms, where only one implication is true: if

$$
X \stackrel{f}{\longrightarrow} Y \stackrel{g}{\longleftarrow} Z
$$

are morphisms in OrdGrp such that $f$ and $g$ are jointly strongly epimorphic in Grp and their restrictions to the positive cones are jointly strongly epimorphic in Mon, then $f$ and $g$ are jointly strongly epimorphic in OrdGrp. Indeed, suppose that $f$ and $g$ factor through a monomorphism 
$m:$

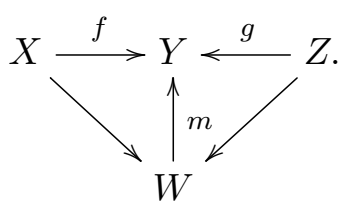

Since $f$ and $g$ are jointly strongly epimorphic in Grp, we can assume that $W=Y$ and that $m=\operatorname{id}_{Y}$. If $P_{W} \neq P_{Y}$, then the restrictions of $f$ and $g$ to the positive cones would factor through a proper inclusion $P_{W} \rightarrow P_{Y}$, and so they would not be jointly strongly epimorphic in Mon.

The converse implication is false, as the following example shows.

Example 2.8. Let $\mathbb{Z}$ be the group of integers with the usual order, namely $P_{\mathbb{Z}}=\mathbb{N}$. Consider the two coproduct inclusions in OrdGrp

$$
\mathbb{Z} \stackrel{\iota_{1}}{\longrightarrow} \mathbb{Z}+\mathbb{Z} \stackrel{\iota_{2}}{\longleftarrow} \mathbb{Z}
$$

$\iota_{1}$ and $\iota_{2}$ are clearly jointly strongly epimorphic in OrdGrp, but their restrictions to the positive cones are not jointly strongly epimorphic in Mon, since the supremum of $\iota_{1}(\mathbb{N})$ and $\iota_{2}(\mathbb{N})$ is $\mathbb{N}+\mathbb{N}$, which is not the positive cone of $\mathbb{Z}+\mathbb{Z}$, because $\mathbb{N}+\mathbb{N}$ is not closed under conjugation in $\mathbb{Z}+\mathbb{Z}$.

Since closure under conjugation holds trivially in the abelian case, it is easily checked that the result is true for abelian groups: when $Y$ is an abelian group, a pair $X \stackrel{f}{\longrightarrow} Y \stackrel{g}{\longleftarrow} Z$ is jointly strongly epimorphic in OrdGrp if and only if both its underlying group homomorphisms in Grp and its restriction to the positive cones in Mon are so.

\section{A SPECIAL ADJUNCTION}

The functor P: OrdGrp $\rightarrow$ Mon, which sends a preordered group to its positive cone, factors through the category $\mathbf{M o n}_{\text {can }}$ of monoids with cancellation: indeed, every submonoid of a group satisfies both left and right cancellation properties. The functor P: OrdGrp $\rightarrow \mathbf{M o n}_{\mathrm{can}}$ has a left adjoint:

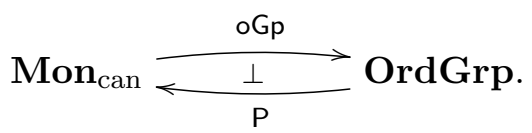

It is obtained in the following way: given a monoid $M$ with cancellation, we consider its group completion $\mathrm{Gp}(M)$, i.e. the quotient of the free group $\mathrm{F}(M)$ on the underlying set of $M$ w.r.t. the normal subgroup generated by the elements of the form $[a]+[b]-[a+b]$ (see $[15,16,17]$ ). Then we equip the group $\operatorname{Gp}(M)$ with the order induced by the positive cone $P_{\mathrm{Gp}(M)}$ which is the smallest submonoid of $\operatorname{Gp}(M)$ containing $\zeta_{M}(M)$ and closed under conjugation, where $\zeta_{M}: M \rightarrow \mathrm{Gp}(M)$ is the unit of the group completion adjunction. This clearly gives rise to a preordered group, and this construction is functorial. Let us check that we actually get an adjunction. Given a monoid $M$ with cancellation and a preordered group $X$, a morphism $f: \mathrm{oGp}(M) \rightarrow X$ restricts to a monoid homomorphism $P_{\mathrm{Gp}(M)} \rightarrow P_{X}$; composing it with the homomorphism $M \rightarrow \zeta_{M}(M) \hookrightarrow P_{\mathrm{Gp}(M)}$, we obtain the desired homomorphism $M \rightarrow P_{X}$. Conversely, given a monoid homomorphism $h: M \rightarrow P_{X}$, we compose it with the inclusion of $P_{X}$ into $X$, thus obtaining a homomorphism from $M$ to the group $X$. By the universal property of the group completion, this determines a unique group homomorphism $f: \operatorname{Gp}(M) \rightarrow X$ which extends $h$. Such $f$ is monotone: indeed, $\zeta_{M}(M)$ is contained in $f^{-1}\left(P_{X}\right)$, and $f^{-1}\left(P_{X}\right)$ is closed under conjugation in $\mathrm{Gp}(M)$. Hence $P_{\mathrm{Gp}(M)}$ is contained in $f^{-1}\left(P_{X}\right)$, and thus $f\left(P_{\mathrm{Gp}(M)}\right) \subseteq P_{X}$. 
So $f: \mathrm{oGp}(M) \rightarrow X$ is a morphism in OrdGrp. It is straightforward to check that these correspondences are natural and inverse to each other.

The previous adjunction restricts to an adjunction between the category OrdAb of preordered abelian groups and the category $\mathbf{C M o n}_{\mathrm{can}}$ of commutative monoids with cancellation:

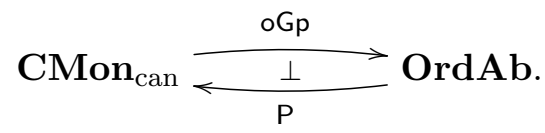

In this case, the left adjoint oGp is much simpler: indeed, the group completion $\mathrm{Gp}(M)$ of a commutative monoid $M$ with cancellation is the quotient of the direct product $M \times M$ w.r.t. the congruence $\sim$ defined by $(a, b) \sim(c, d)$ if and only if $a+d=b+c$. Moreover, $M$ is a submonoid of $\mathrm{Gp}(M)$ (since $M$ is a monoid with cancellation) and, being $\mathrm{Gp}(M)$ abelian, $M$ is closed under conjugation in it, hence it is the positive cone of a compatible preorder on $\operatorname{Gp}(M)$. Thus, the composite functor P.oGp is isomorphic to the identity functor on $\mathbf{C M o n}_{\mathrm{can}}$. In other terms, the unit $\eta$ of this adjunction is an isomorphism. Observe that the functor $\mathrm{P}$ is not faithful: given any two abelian groups $X$ and $Y$, equipped with the trivial order (i.e. $P_{X}=P_{Y}=0$ ), then any group homomorphism from $X$ to $Y$ is monotone and it is sent by $\mathrm{P}$ to the trivial map $0 \rightarrow 0$.

For the larger adjunction (A), it is not true that the unit is an isomorphism. In fact, the situation is much more complicated. Clearly, being embeddable in a group is a necessary condition on a monoid $M$ to have an isomorphism as the unit of the adjunction (A), and being cancellative is not enough for a monoid to be embeddable in a group. Mal'tsev gave in $[16,17]$ a characterization of embeddable monoids (see also Chapter VII of [12] for a description of such monoids). Here we are only interested in the study of those embeddable monoids.

Proposition 3.1. If a monoid $M$ is the positive cone of a preordered group, then $M=P_{\mathrm{oGp}(M)}$. Proof. We start by observing that, if a monoid is embeddable in a group, then it is embeddable in its group completion. Consider then the following commutative diagram of monoid homomorphisms:

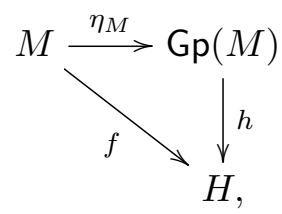

where $H$ is a group and $f$ is injective. Then $h$ is injective, too. Indeed, since $h$ is injective when restricted to $M$ and a generic element of $\mathrm{Gp}(M)$ is an equivalence class of chains of the form $m_{1}-m_{2}+m_{3}+\ldots-m_{k}$, injectivity of $h$ follows by induction on the length of the chain.

Now, if $M=P_{H}$ for a preordered group $H$, then the injective morphism $h$ : oGp $(M) \rightarrow H$ restricts to an injection $P_{\mathrm{oGp}(M)} \rightarrow M=P_{H}$. Since $M \subseteq P_{\mathrm{oGp}(M)}$, the proof is complete.

Proposition 3.2. Given a monoid $M$ which is embeddable in a group, the component $\eta_{M}: M \rightarrow$ $P_{\mathrm{oGp}(M)}$ of the unit of the adjunction (A) is an isomorphism if and only if the following condition is satisfied:

$$
\forall a, b \in M \quad \exists x, y \in M \quad \text { such that } \quad a+b=b+x=y+a .
$$

Proof. If $M$ is the positive cone of oGp $(M)$, then it is a submonoid closed under conjugation in $\mathrm{Gp}(M)$. Hence, for all $a, b \in M$ the elements

$$
y=a+b-a \quad \text { and } \quad x=-b+a+b
$$


belong to $M$, and this gives Condition (B). Conversely, to prove that $M$ is closed under conjugation in $\operatorname{Gp}(M)$, it suffices to conjugate an element $a \in M$ with elements of the form $b$ or $-b$, with $b \in M$, because all elements in $\operatorname{Gp}(M)$ are chains of such. Now, supposing that Condition (B) holds, we have:

$$
a+b=b+x \Rightarrow-b+a+b=x \in M ; \quad a+b=y+a \Rightarrow a+b-a=y \in M,
$$

and this concludes the proof.

The previous proposition actually gives a characterization of the monoids that are positive cones of a preordered group. Indeed, we have the following

Corollary 3.3. A monoid $M$ which is embeddable in a group is the positive cone of a preordered group if and only if Condition (B) holds.

\section{Algebraic properties of OrdGrp}

Since a morphism in OrdGrp is an epimorphism if and only if it is surjective, hence if and only if it is an epimorphism in Grp, we have that OrdGrp is a weakly Mal'tsev category [18], which means that for every pullback of split epimorphisms as in the following diagram

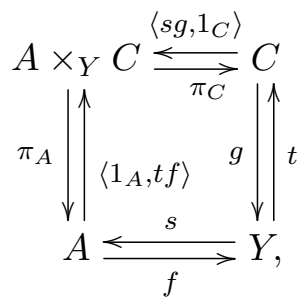

the morphisms $\left\langle 1_{A}, t f\right\rangle$ and $\left\langle s g, 1_{C}\right\rangle$ are jointly epimorphic. In fact, this is a particular instance of a more general context which is considered in [19, 20].

Actually, we have more: OrdGrp is weakly protomodular, which means that, for every split epimorphism $A \underset{f}{\stackrel{s}{\longrightarrow}} B$ with kernel $k: X \rightarrow A, k$ and $s$ are jointly epimorphic. The proof that a weakly protomodular category is weakly Mal'tsev is essentially the same as the proof that a protomodular category is Mal'tsev (see, for example, [2]). Moreover, this explains why OrdGrp is a normal category: indeed, as shown in [8], a weakly protomodular category is normal.

\section{Furthermore}

Proposition 4.1. OrdGrp is a unital category [6], i.e. for any two objects $X$ and $Y$ the canonical morphisms $\langle 1,0\rangle: X \rightarrow X \times Y$ and $\langle 0,1\rangle: Y \rightarrow X \times Y$ are jointly strongly epimorphic.

Proof. Given $X, Y, Z \in$ OrdGrp, consider the following commutative diagram

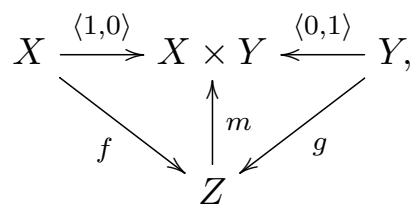

where $m$ is a monomorphism. Being Grp unital, $m$ is an isomorphism of groups; it only remains to show that its inverse $t$ is monotone. Suppose that $(x, y) \leq\left(x^{\prime}, y^{\prime}\right)$; then $x \leq x^{\prime}$ and $y \leq y^{\prime}$, which implies that $f(x) \leq f\left(x^{\prime}\right)$ and $g(y) \leq g\left(y^{\prime}\right)$. But then

$$
t(x, y)=f(x)+g(y) \leq f\left(x^{\prime}\right)+g\left(y^{\prime}\right)=t\left(x^{\prime}, y^{\prime}\right) .
$$


Proposition 4.2. OrdAb has biproducts.

Proof. We want to show that, for any $X, Y \in$ OrdAb,

$$
X \stackrel{\langle 1,0\rangle}{\longrightarrow} X \times Y \stackrel{\langle 0,1\rangle}{\longleftarrow} Y
$$

is a coproduct diagram. Given morphisms $f: X \rightarrow Z$ and $g: Y \rightarrow Z$, the (necessarily unique, by unitality) morphism $\alpha$ which makes the following diagram commute:

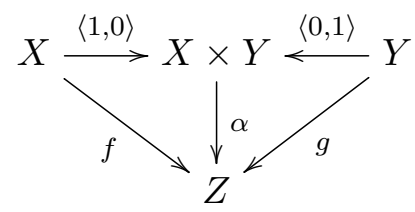

is just the composite $X \times Y \stackrel{f \times g}{\longrightarrow} Z \times Z \stackrel{m_{Z}}{\longrightarrow} Z$, where $m_{Z}$ is the group operation of $Z$.

The categories OrdGrp and OrdAb are not protomodular [5]. Indeed, if $\mathbb{Z}$ is the group of integers with the usual order, we can equip the direct product $\mathbb{Z} \times \mathbb{Z}$ with (at least) two preorders: the product one, given by

$$
(x, y) \leq\left(x^{\prime}, y^{\prime}\right) \Leftrightarrow x \leq x^{\prime} \text { and } y \leq y^{\prime}
$$

and the (reverse) lexicographical one, given by

$$
(x, y) \leq\left(x^{\prime}, y^{\prime}\right) \Leftrightarrow y<y^{\prime} \quad \text { or } \quad y=y^{\prime}, x \leq x^{\prime}
$$

Denoting by $\mathbb{Z} \times_{p} \mathbb{Z}$ and $\mathbb{Z} \times_{l} \mathbb{Z}$ the two corresponding ordered groups, we obtain a morphism of split extensions in OrdGrp:

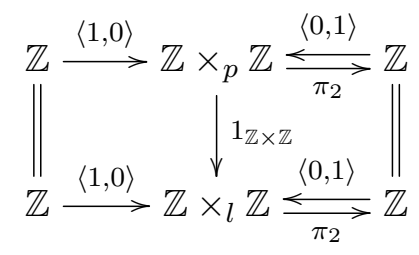

whose middle component is not an isomorphism, because its inverse is not monotone. So the Split Short Five Lemma does not hold in OrdGrp, nor in OrdAb.

In order to describe local categorical-algebraic properties of "good" objects in categories that are not protomodular or Mal'tsev, in [22] some notions have been considered for objects. In order to recall them, we first need the following

Definition 4.3. A point (i.e. a split epimorphism with a fixed section) $A \underset{f}{\stackrel{s}{\leftrightarrows}} B$ with kernel $k: X \rightarrow A$ in a pointed finitely complete category is strong if $k$ and $s$ are jointly strongly epimorphic. It is stably strong if every pullback of it along any morphism $g: C \rightarrow B$ is strong.

Definition $4.4([22])$. An object $Y$ of a finitely complete category $\mathbf{C}$ is

(1) a strongly unital object if the point $Y \times Y \underset{\pi_{2}}{\stackrel{\langle 1,1\rangle}{\longrightarrow}} Y$ is stably strong; 
(2) a Mal'tsev object if, for every pullback of split epimorphisms over $Y$ as in the following diagram

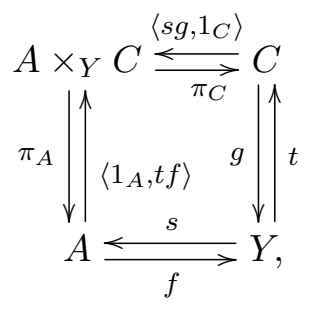

the morphisms $\left\langle 1_{A}, t f\right\rangle$ and $\left\langle s g, 1_{C}\right\rangle$ are jointly strongly epimorphic;

(3) a protomodular object if every point over $Y$ is stably strong.

We observe that, in a unital category, an object is strongly unital if, and only if, it is gregarious in the sense of [2], Definition 1.9.1. Proposition 1.9.2 in [2] gives a characterization of gregarious (= strongly unital) objects in Mon: a monoid $M$ is gregarious if and only if for any $m$ in $M$ there exist $u, v \in M$ such that $u+m+v=0$. Every group is a gregarious monoid, of course, but there are gregarious monoids that are not groups, as Counterexample 1.9.3 in [2] shows. However, a crucial fact for our study of "group-like" objects in OrdGrp is the following

Lemma 4.5. If a monoid $M$ is the positive cone of a preordered group, then it is gregarious if, and only if, it is a group.

Proof. Suppose that, for any $m$ in $M$, there exist $u, v \in M$ such that $u+m+v=0$. Thanks to Corollary 3.3, we know that there exists $y \in M$ such that $m+v=y+m$. Then $u+y+m=0$ and $u+y$ is a left inverse for $m$. Every element having a left inverse, $M$ is a group.

Now we can state and prove the main result of this section:

Theorem 4.6. For a preordered group $Y$, the following conditions are equivalent:

(i) $Y$ is protomodular;

(ii) $Y$ is a Mal'tsev object;

(iii) $Y$ is strongly unital;

(iv) $P_{Y}$ is a group, i.e. the preorder relation on $Y$ is an equivalence relation.

Proof. (iv) $\Rightarrow$ (i): given a diagram in OrdGrp as

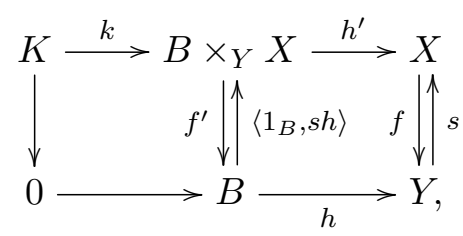

being Grp a protomodular category, we already know that $k$ and $\left\langle 1_{B}, s h\right\rangle$ are jointly strongly epimorphic in Grp. So, thanks to Remark 2.7, it suffices to show that their restrictions to the positive cones are jointly strongly epimorphic in Mon. Consider then the following diagram:

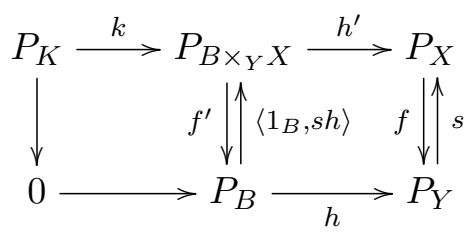

(using the same notations for the restrictions to the positive cones). The functor $\mathrm{P}$ preserves limits, hence the two downward squares are still pullbacks. Since $P_{Y}$ is a group, it is a protomodular object in Mon ([22], Theorem 7.7). Then the thesis follows. 
(i) $\Rightarrow$ (ii) follows from Proposition 7.2 in [22].

(ii) $\Rightarrow$ (iii) follows from Proposition 6.3 in [22], being OrdGrp a regular category.

(iii) $\Rightarrow$ (iv): thanks to Lemma 4.5 , it suffices to prove that $P_{Y}$ is a gregarious monoid. Suppose that there is an element $b \in P_{Y}$ for which there are no $u, v \in M$ with $u+b+v=0$. Let $X=\langle b\rangle$ be the subgroup of $Y$ generated by $b$, with the induced preorder, and $j: X \hookrightarrow Y$ the inclusion. Observe that, for $n \in \mathbb{N}, n b=0$ if and only if $n=0$ (otherwise there would exist $u, v \in M$ with $u+b+v=0)$. Hence $X$, as a preordered group, is isomorphic to $\mathbb{Z}$ with its usual order, namely $P_{X}=\{n b \mid n \in \mathbb{N}\}$. Consider then the following right-hand side pullback in OrdGrp:

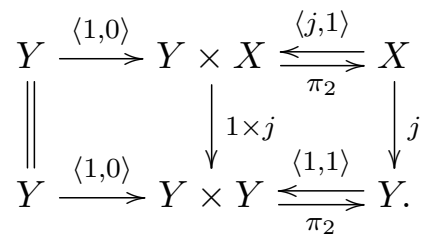

We are going to prove that the positive cone of $Y \times X$ is not the positive cone $P$ generated by

$$
C=\left\{(c, 0) \mid c \in P_{Y}\right\} \cup\{(n b, n b) \mid n \in \mathbb{N}\} .
$$

To do that, we show that $(0, b) \notin P$. The positive cone $P$ is obtained by iteration of two steps, using addition and conjugation. Formally, let $C(0)=A(0)=C$, and define, for $k \in \mathbb{N}$,

$$
\begin{gathered}
A(k+1)=\{s+t \mid s, t \in C(k)\}, \\
C(k+1)=\{w+s-w \mid s \in A(k+1), w \in Y \times X\} .
\end{gathered}
$$

Then $P=\bigcup_{k \in \mathbb{N}} A(k)=\bigcup_{k \in \mathbb{N}} C(k)$. Now we show, by induction, that if $(c, b) \in P$, then

$$
c=y+u+b+v-y, \quad \text { for some } \quad y \in Y, u, v \in P_{Y} .
$$

This is true for $(c, b) \in C(0)$. If it is true for $(c, b) \in C(k)$, then:

- if $(a, b) \in A(k+1)$, i.e.

$$
(a, b)=\left(c_{1}, b_{1}\right)+\left(c_{2}, b_{2}\right)
$$

for some $\left(c_{1}, b_{1}\right),\left(c_{2}, b_{2}\right) \in C(k)$, then either $b_{1}=b$ or $b_{2}=b$. Suppose $b_{1}=b$. Then

$$
a=y+a_{1}+b+a_{2}-y+c_{2}=y+a_{1}+b+a_{2}+\left(-y+c_{2}+y\right)-y,
$$

with $-y+c_{2}+y \in P_{Y}$. Hence $(a, b)$ satisfies our claim. The case $b_{2}=b$ is analogous.

- if $(a, b) \in C(k+1)$, i.e. $a=z+c-z$ with $(c, b) \in A(k+1)$, then

$$
a=z+y+a_{1}+b+a_{2}-y-z,
$$

and our claim is proved. Now, if the point $Y \times X \underset{\pi_{2}}{\stackrel{\langle j, 1\rangle}{\lessgtr}} X$ is strong, then there is some $k \in \mathbb{N}$ such that $(0, b) \in A(k)$, and then, since

$$
0=y+a_{1}+b+a_{2}-y,
$$

with $y \in Y, a_{1}, a_{2} \in P_{Y}$, we would get $a_{1}+b+a_{2}=0$, which is a contradiction.

The previous theorem shows, in particular, that OrdGrp is not a Mal'tsev category nor a strongly unital one, since a finitely complete category is Mal'tsev (resp. strongly unital) if and only if every object is Mal'tsev (resp. strongly unital); see [22].

We conclude this section by observing that, since the full subcategory of protomodular objects in OrdGrp, i.e. the full subcategory whose objects are the preordered groups whose positive 
cone is a group, is closed under finite limits in OrdGrp, it is a protomodular category, thanks to Corollary 7.4 in [22]. It is actually the category of the preordered groups such that the inverse map is monotone, hence it is the category $\operatorname{Grp}(\mathbf{O r d})$ of internal groups in Ord.

\section{A study of Split extensions in OrdGrp}

In order to understand better the protomodular aspects of OrdGrp, we will analyse the structure of split extensions in this category.

Let $X \stackrel{k}{\longrightarrow} A \underset{p}{\stackrel{s}{\longrightarrow}} B$ be a split extension in OrdGrp. Being also a split extension in Grp, we have that $A$, as a group, is isomorphic to the semidirect product $X \rtimes_{\varphi} B$ w.r.t. the action $\varphi$ of $B$ on $X$ given by $\varphi_{b}(x)=k^{-1}(s(b)+k(x)-s(b))$; more precisely, in Grp the given split extension is isomorphic to the split extension $X \stackrel{\langle 1,0\rangle}{\longrightarrow} X \rtimes_{\varphi} B \underset{\pi_{B}}{\stackrel{\langle 0,1\rangle}{\longrightarrow}} B$. In addition, if we consider the corresponding group action $\varphi: B \rightarrow \operatorname{Aut}(X)$ described above, we obtain that

$$
\varphi_{b}: X \rightarrow X \text { is monotone for every } b \in B,
$$

because the positive cone of $A$ is closed under conjugation.

Throughout this section, for a given split extension

$$
X \stackrel{\langle 1,0\rangle}{\longrightarrow} X \rtimes_{\varphi} B \stackrel{\langle 0,1\rangle}{\stackrel{\pi_{B}}{\longrightarrow}} B
$$

in Grp, with $X$ and $B$ preordered groups and $\varphi_{b}: X \rightarrow X$ monotone for every $b \in B$, we will study the compatible preorders on $X \rtimes_{\varphi} B$, in the sense that they make $X \rtimes_{\varphi} B$ a preordered group and (D) a split extension in OrdGrp.

There are two possible "canonical" preorders on $X \rtimes_{\varphi} B$, namely:

- the product preorder, whose positive cone is $P_{\text {prod }}=P_{X} \times P_{B}$,

- and the (reverse) lexicographical preorder, which is defined by:

$$
(x, b) \leq\left(x^{\prime}, b^{\prime}\right) \Leftrightarrow b<b^{\prime} \text { or }\left(b=b^{\prime} \text { and } x \leq x^{\prime}\right) .
$$

In other terms, the positive cone of the lexicographical preorder is

$$
P_{\text {lex }}=\left\{(x, b) \in X \rtimes_{\varphi} B \mid b>0 \text { or }(b=0 \text { and } x \geq 0)\right\} ;
$$

indeed $(x, b) \leq\left(x^{\prime}, b^{\prime}\right)$ with respect to the preorder induced by the positive cone $P_{\text {lex }}$ if, and only if,

$$
\left(x^{\prime}, b^{\prime}\right)-(x, b)=\left(x^{\prime}, b^{\prime}\right)+\left(-\varphi_{-b}(x),-b\right)=\left(x^{\prime}-\varphi_{b^{\prime}-b}(x), b^{\prime}-b\right) \in P_{\mathrm{lex}},
$$

which means either $b<b^{\prime}$ or $b=b^{\prime}$ and $x^{\prime}-x \geq 0$, i.e. $x \leq x^{\prime}$.

As a first example consider the ordered group $\mathbb{Z}$ and the split extension $\mathbb{Z} \stackrel{\langle 1,0\rangle}{\longrightarrow} \mathbb{Z} \times \mathbb{Z} \underset{\pi_{2}}{\stackrel{\langle 0,1\rangle}{\longrightarrow}} \mathbb{Z}$. As we mentioned before, both the product the lexicographic preorders are compatible. We will see in Example 5.8 that these are not the only compatible preorders.

However, in general the two preorders mentioned above are not always compatible. Before giving some necessary and sufficient conditions for their compatibility, we observe they are the lower and the upper bound, respectively, for every compatible preorder on $X \rtimes_{\varphi} B$ : 
Proposition 5.1. If $P$ is the positive cone of a compatible preorder on $X \rtimes_{\varphi} B$, then:

$$
P_{\text {prod }} \subseteq P \subseteq P_{\text {lex }}
$$

Proof. If $x \leq x^{\prime}$ and $b \leq b^{\prime}$, then $(x, 0) \leq\left(x^{\prime}, 0\right)$ and $(0, b) \leq\left(0, b^{\prime}\right)$, because $\langle 1,0\rangle$ and $\langle 0,1\rangle$ are monotone maps. Thus

$$
(x, b)=(x, 0)+(0, b) \leq\left(x^{\prime}, 0\right)+\left(0, b^{\prime}\right)=\left(x^{\prime}, b^{\prime}\right) .
$$

This proves that $P_{X} \times P_{B} \subseteq P$. Moreover, if $(x, b) \leq\left(x^{\prime}, b\right)$ in $X \rtimes_{\varphi} B$, then $(x, b)-(0, b) \leq$ $\left(x^{\prime}, b\right)-(0, b)$, hence $(x, 0) \leq\left(x^{\prime}, 0\right)$. Being $\langle 1,0\rangle: X \rightarrow X \rtimes_{\varphi} B$ a kernel in OrdGrp, this implies that $x \leq x^{\prime}$. This shows that $P \subseteq P_{\text {lex }}$.

Now we give necessary and sufficient conditions for the product and the lexicographical preorders to be compatible. We start with the product preorder.

Proposition 5.2. The product preorder is compatible if and only if:

$$
\forall b \in P_{B}, \forall x \in X \quad \varphi_{b}(x) \geq x .
$$

Proof. $P_{X} \times P_{B}$ is a positive cone if and only if, for every $\left(x_{1}, b_{1}\right),\left(x_{2}, b_{2}\right) \in P_{X} \times P_{B}$ and every $(x, b) \in X \rtimes_{\varphi} B$, the elements

$$
\left(x_{2}, b_{2}\right)+\left(x_{1}, b_{1}\right) \text { and }(x, b)+\left(x_{1}, b_{1}\right)-(x, b)
$$

belong to $P_{X} \times P_{B}$. Since

$$
\left(x_{2}, b_{2}\right)+\left(x_{1}, b_{1}\right)=\left(x_{2}+\varphi_{b_{2}}\left(x_{1}\right), b_{2}+b_{1}\right) \in P_{X} \times P_{B},
$$

due to monotonicity of $\varphi_{b_{2}}$, and

$$
(x, b)+\left(x_{1}, b_{1}\right)-(x, b)=\left(x+\varphi_{b}\left(x_{1}\right)+\varphi_{b+b_{1}-b}(-x), b+b_{1}-b\right),
$$

we have that $P_{X} \times P_{B}$ is a positive cone if and only if:

$$
\forall x \in X, \forall x_{1} \in P_{X}, \forall b \in B, \forall b_{1} \in P_{B}, x+\varphi_{b}\left(x_{1}\right)+\varphi_{b+b_{1}-b}(-x) \in P_{X} .
$$

Since $b+b_{1}-b \in P_{B}$ (being $P_{B}$ closed under conjugation) and $\varphi_{b}\left(x_{1}\right) \in P_{X}$ (because $\varphi_{b}$ is monotone), it is immediate to see that, under (E), Condition (F) is satisfied. Conversely, assuming $(\mathrm{F})$ and choosing $b=0$ and $x_{1}=0$ there, we get that $x+\varphi_{b_{1}}(-x) \in P_{X}$, i.e. $\varphi_{b_{1}}(-x) \geq-x$ for all $x \in X$ and $b_{1} \in P_{B}$, which is clearly equivalent to (E).

Remark 5.3. It is immediate to see that the product preorder on a semidirect product $X \rtimes_{\varphi} B$ is compatible if and only if the product projection $\pi_{X}: X \rtimes_{\varphi} B \rightarrow X$ is a monotone map. We recall that $\pi_{X}$ is a group homomorphism if and only if the action $\varphi$ is trivial, i.e. the semidirect product is actually a direct product. In this case, the product preorder is clearly compatible.

Concerning the lexicographical preorder, we have the following:

Proposition 5.4. (1) If the preorder on $B$ is antisymmetric, then the lexicographical preorder on $X \rtimes_{\varphi} B$ is compatible.

(2) If $X$ has a non-positive element, then the lexicographical preorder on $X \rtimes_{\varphi} B$ is compatible if, and only if, the preorder on $B$ is antisymmetric.

Proof. We first show that the preorder on $B$ is antisymmetric if, and only if, given $b, b^{\prime} \in P_{B}$, $b>0$ implies $b+b^{\prime}>0$. Indeed, supposing that $P_{B}$ has no non-trivial invertible elements, if $b+b^{\prime}=0$, then $b^{\prime}=-b$, and since $b^{\prime} \in P_{B}$, this would give $b^{\prime}=0$, and so $b=0$. Conversely, 
if the preorder on $B$ is not antisymmetric, then there exists $b \in P_{B}, b \neq 0$, such that $-b \in P_{B}$. Choosing $b^{\prime}=-b$ we get that $b>0$ but $b+b^{\prime}=0$.

Now suppose that the preorder on $B$ is antisymmetric. We recall that the positive cone of the lexicographical preorder is:

$$
P_{\text {lex }}=\left\{(x, b) \in X \rtimes_{\varphi} B \mid b>0 \text { or }(b=0 \text { and } x \geq 0)\right\} .
$$

Clearly $(0,0) \in P_{\text {lex }}$. If $(x, b),\left(x^{\prime}, b^{\prime}\right) \in P_{\text {lex }}$, then

$$
(x, b)+\left(x^{\prime}, b^{\prime}\right)=\left(x+\varphi_{b}\left(x^{\prime}\right), b+b^{\prime}\right) \in P_{\text {lex }},
$$

since in the case $b>0$ or $b^{\prime}>0$ we have that $b+b^{\prime}>0$, while in the case $b=b^{\prime}=0$ we have $x+\varphi_{b}\left(x^{\prime}\right)=x+x^{\prime} \geq 0$. Finally, if $(x, b) \in X \rtimes_{\varphi} B$ and $\left(x^{\prime}, b^{\prime}\right) \in P_{\text {lex }}$, then

$$
(x, b)+\left(x^{\prime}, b^{\prime}\right)-(x, b)=\left(x+\varphi_{b}\left(x^{\prime}\right)-\left(\varphi_{b+b^{\prime}-b}(x)\right), b+b^{\prime}-b\right) \in P_{\text {lex }},
$$

because $b^{\prime}>0$ implies $b+b^{\prime}-b>0$, while from $b^{\prime}=0$ we get that $\left(x+\varphi_{b}\left(x^{\prime}\right)-x, 0\right) \in P_{\text {lex }}$, since $0=\varphi_{b}(0) \leq \varphi_{b}\left(x^{\prime}\right)$ by monotonicity of $\varphi_{b}$, and $P_{X}$ is closed under conjugation in $X$.

Finally, suppose that there exists $x \in X \backslash P_{X}$. If the preorder on $B$ is not antisymmetric, there is $b \in P_{B}, b \neq 0$, such that $-b \in P_{B}$. Then $(x, b)$ and $(0,-b)$ both belong to $P_{\text {lex }}$, but $(x, b)+(0,-b)=(x, 0)$ does not.

In general, there is no compatible preorder on $X \rtimes_{\varphi} B$. In order to give a concrete counterexample, we first observe that, when $P_{B}$ is a group, there is at most one compatible preorder:

Proposition 5.5. If $P_{B}$ is a group then there is at most one preorder structure on $X \rtimes_{\varphi} B$ making (D) a split extension in OrdGrp.

Proof. Saying that $P_{B}$ is a group is the same as saying that $B$ is a protomodular object in OrdGrp. So, supposing that there are two compatible preorder structures on $X \rtimes_{\varphi} B$, whose positive cones are $P$ and $P^{\prime}$, we get that the intersection $P \cap P^{\prime}$ is a positive cone of a compatible preorder structure, and it is contained in both. Hence we get two monomorphisms $i:\left(X \rtimes_{\varphi} B, P \cap\right.$ $\left.P^{\prime}\right) \rightarrow\left(X \rtimes_{\varphi} B, P\right)$ and $i^{\prime}:\left(X \rtimes_{\varphi} B, P \cap P^{\prime}\right) \rightarrow\left(X \rtimes_{\varphi} B, P^{\prime}\right)$ through which $\langle 1,0\rangle$ and $\langle 0,1\rangle$ factor:
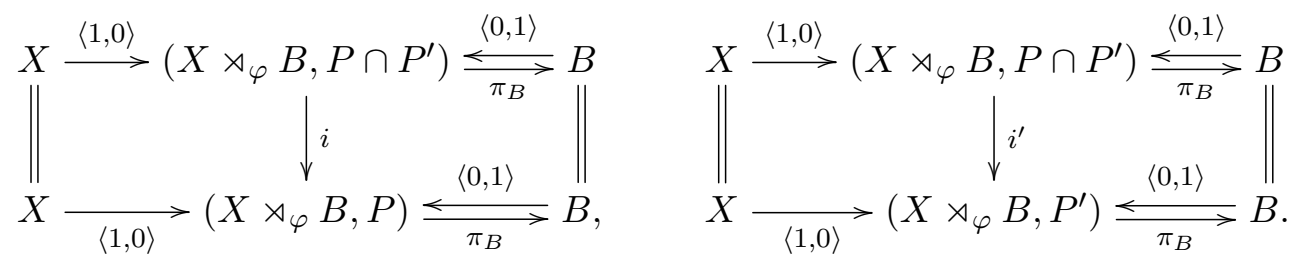

But, being $B$ protomodular, $\langle 1,0\rangle$ and $\langle 0,1\rangle$ are jointly strongly epimorphic, and so $i$ and $i^{\prime}$ are isomorphisms in OrdGrp. This means that $P \cap P^{\prime}=P=P^{\prime}$.

We can actually say more: the unique possible preorder structure is the product one:

Proposition 5.6. If $P_{B}$ is a group the unique possibly compatible preorder structure on $X \rtimes_{\varphi} B$ is the product preorder.

Proof. If $P$ is a positive cone for $X \rtimes_{\varphi} B$ and $(x, b) \in P$, then $b=\pi_{B}(x, b) \in P_{B}$. But $P_{B}$ is a group, hence $-b \in P_{B}$, which implies that $(0,-b) \in P$. Hence

$$
(x, 0)=(x, b)+(0,-b) \in P .
$$


Since $\langle 1,0\rangle$ is the kernel of $\pi_{B}$, this implies that $x \in P_{X}$, i.e. $(x, b) \in P_{X} \times P_{B}$. So, $P$ is contained in the positive cone of the product preorder. But we proved that the product preorder is the smallest possible one, so $P=P_{X} \times P_{B}$.

Hence, if we consider a protomodular object $B$ and a group action of $B$ on $X$ which does not satisfy the (necessary and sufficient) condition for the product preorder to be compatible, then there is no compatible preorder structure on the semidirect product $X \rtimes_{\varphi} B$. The following is a concrete example:

Example 5.7. Consider the split extension $X \stackrel{\langle 1,0\rangle}{\longrightarrow} X \rtimes_{\varphi} B \underset{\pi_{B}}{\stackrel{\langle 0,1\rangle}{\longrightarrow}} B$, where $X=\mathbb{Z}$ with the identity preorder (i.e. $\left.P_{X}=\{0\}\right), B=\mathbb{Z}$ with the total preorder (i.e. $P_{B}=\mathbb{Z}$ ), and the action $\varphi$ is given by $\varphi_{b}(x)=(-1)^{b} x$. Then the product preorder is not compatible, because $\varphi_{1}(x)=-x \nsupseteq x$ if $x \neq 0$.

Looking at the opposite situation, namely when the relation on $B$ is antisymmetric, we can get many compatible structures on the semidirect product, as the following example shows.

Example 5.8. Consider the split extension $\mathbb{Z} \stackrel{\langle 1,0\rangle}{\longrightarrow} \mathbb{Z} \times \mathbb{Z} \underset{\pi_{2}}{\stackrel{\langle 0,1\rangle}{\leftrightarrows}} \mathbb{Z}$, where on both copies of $\mathbb{Z}$ there is the usual order (namely $P_{\mathbb{Z}}=\mathbb{N}$ ). Then there is a bijection between the set of positive cones of the compatible preorders on $\mathbb{Z} \times \mathbb{Z}$ and the set of sequences $\left(x_{n}\right)_{n \in \mathbb{N}}$ in $\mathbb{N} \cup\{\infty\}$ such that $x_{0}=0$ and, for all $n, m \in \mathbb{N}, x_{n+m} \geq x_{n}+x_{m}$.

Indeed, for every positive cone $P$, we can define the sequence $\left(x_{n}\right)$, where

$$
x_{0}=0, \quad x_{n}=\sup \{k \in \mathbb{N} \mid(-k, n) \in P\} \text { for } n>0 .
$$

If $(-k, n)$ and $\left(-k^{\prime}, m\right)$ belong to $P$, then $\left(-\left(k+k^{\prime}\right), n+m\right) \in P$, from which we get that $x_{n+m} \geq x_{n}+x_{m}$. Conversely, given a sequence $\left(x_{n}\right)$ with $x_{0}=0$ and, for all $n, m \in \mathbb{N}$, $x_{n+m} \geq x_{n}+x_{m}$, we define

$$
P=\left\{(-k, n) \mid n \in \mathbb{N}, k \in \mathbb{Z}, k \leq x_{n}\right\} .
$$

It is clear that $P_{\text {prod }} \subseteq P \subseteq P_{\text {lex }}$; let us check that $P$ is a submonoid of $\mathbb{Z} \times \mathbb{Z}$ (closedness under conjugation comes for free, since all groups involved here are abelian):

$(-k, n),\left(-k^{\prime}, m\right) \in P \Rightarrow k \leq x_{n}, k^{\prime} \leq x_{m} \Rightarrow-\left(k+k^{\prime}\right) \leq x_{n}+x_{m} \leq x_{n+m} \Rightarrow\left(-\left(k+k^{\prime}\right), n+m\right) \in P$.

Thanks to this bijection, we can see that the set of compatible preorders is uncountable. In fact, the set of sequences $\left(x_{n}\right)$ as above such that the value $\infty$ is never reached is uncountable. To see this, suppose that this set is countable. So we get a sequence of sequences:

$$
\left(x_{n}^{1}\right), \ldots,\left(x_{n}^{k}\right), \ldots
$$

Consider the sequence $\left(y_{n}\right)$ defined by:

$$
\begin{aligned}
& y_{0}=0 ; \\
& y_{1}=x_{1}^{1}+1 ; \\
& y_{2}=\max \left\{x_{2}^{2}+1, y_{1}+y_{1}\right\} ; \\
& y_{3}=\max \left\{x_{3}^{3}+1, y_{i}+y_{j}: i+j=3\right\} ; \\
& y_{n}=\max \left\{x_{n}^{n}+1, y_{i}+y_{j}: i+j=n\right\} .
\end{aligned}
$$


It is immediate to see that $\left(y_{n}\right)$ satisfies the conditions to define a positive cone, that it never reaches the value $\infty$, and that it is different from all sequences $\left(x_{n}^{k}\right)$, because, for all $n \in \mathbb{N}$, $y_{n} \neq x_{n}^{n}$. This contradicts the hypothesis that the set is countable.

\section{Relative protomodularity}

In Section 4 we used the objectwise approach of [22] to describe what remains of protomodularity in the category OrdGrp. In $[9,10]$ a different approach was considered with a similar scope: describing partial homological properties of "weak" algebraic categories, having as guiding example the category Mon of monoids. This alternative approach does not try to identify "good" objects, but rather a "good" class of points, relatively to which the category has strong homological properties, similar to the ones of protomodular categories. The aim of this section is to study the category OrdGrp from this alternative point of view. We will see that there are at least two good classes of points we can consider. For both, we will identify a protomodular subcategory of OrdGrp, called the protomodular core relatively to the chosen class of points. Not so surprisingly, in both cases the protomodular core will be the full subcategory of OrdGrp whose objects are the preordered groups whose positive cone is a group. This gives a strong parallelism (which does not hold, in general) between the objectwise approach of Section 4 and the one relative to classes of points.

We start by recalling from [10] the following

Definition 6.1. Let $\mathbf{C}$ be a pointed finitely complete category, and $S$ a class of points in $\mathbf{C}$ which is stable under pullbacks and closed in $\operatorname{Pt}(\mathbf{C})$ under finite limits. C is $S$-protomodular if every point in $S$ is a strong point.

The main example of an $S$-protomodular category is the category Mon of monoids w.r.t. the class $S$ of Schreier points [21]: a point

$$
X \stackrel{k}{\longrightarrow} A \underset{p}{\stackrel{s}{\longrightarrow}} B
$$

in Mon is a Schreier point if, for every $a \in A$, there exists a unique $x \in X$ such that $a=k(x)+s f(a)$. This happens if and only if there exists a unique map $q: A \rightarrow X$ (which is not a monoid homomorphism, in general) such that $a=k q(a)+s f(a)$ for all $a \in A$. As explained in [21], Schreier points correspond to classical monoid actions, where an action of a monoid $B$ on a monoid $X$ is a monoid homomorphism $B \rightarrow \operatorname{End}(X)$, where $\operatorname{End}(X)$ is the monoid of endomorphisms of $X$.

We observe that, given a split extension $X \stackrel{\langle 1,0\rangle}{\longrightarrow} X \rtimes_{\varphi} B \underset{\pi_{B}}{\stackrel{\langle 0,1\rangle}{\longrightarrow}} B$ in Grp, where $X$ and $B$ are preordered groups and the action $\varphi$ satisfies Condition (E), the action $\varphi$ restricts to a monoid homomorphism $\varphi_{\mid P_{B}}: P_{B} \rightarrow \operatorname{End}\left(P_{X}\right)$, i.e. to a monoid action of $P_{B}$ on $P_{X}$. This means that, when the semidirect product $X \rtimes_{\varphi} B$ is equipped with the product preorder, the restriction of the split extension $X \stackrel{\langle 1,0\rangle}{\longrightarrow} X \rtimes_{\varphi} B \underset{\pi_{B}}{\stackrel{\langle 0,1\rangle}{\longrightarrow}} B$ to the positive cones is a Schreier split extension of monoids. The converse is also true: if the split extension $X \stackrel{\langle 1,0\rangle}{\longrightarrow} X \rtimes_{\varphi} B \stackrel{\langle 0,1\rangle}{\underset{\pi_{B}}{\longrightarrow}} B$, restricted to the positive cones, is a Schreier split extension of monoids, then, as a set, the positive cone $P_{X \rtimes_{\varphi} B}$ is the cartesian product $P_{X} \times P_{B}$, which says that the preorder on $X \rtimes_{\varphi} B$ is the product 
preorder. This fact allows us to show that OrdGrp is an $S$-protomodular category w.r.t. a suitable class $S$ of points.

Proposition 6.2. Let $S$ be the class of points $X \stackrel{k}{\longrightarrow} A \underset{p}{\stackrel{s}{\leftrightarrows}} B$ in OrdGrp whose restriction to the positive cones is a Schreier point in Mon. Then OrdGrp is S-protomodular.

Proof. We already observed that, if two morphisms in OrdGrp are jointly strongly epimorphic in Grp and their restrictions to the positive cones are jointly strongly epimorphic in Mon, then they are jointly strongly epimorphic in OrdGrp. Grp is a protomodular category, so every point in it is strong. Then the thesis follows, observing that both the forgetful functor OrdGrp $\rightarrow$ Grp and the positive cone functor $P:$ OrdGrp $\rightarrow$ Mon preserve limits.

We observe that the class $S$ we are considering in OrdGrp is the class of points (G) such that the map $q: A \rightarrow X$ defined by $q(a)=k^{-1}(a-s f(a))$ is monotone. Indeed, this map $q$ corresponds, up to isomorphism, to the product projection $\pi_{X}: X \rtimes_{\varphi} B \rightarrow X$, which is monotone if and only if the preorder on $X \rtimes_{\varphi} B$ is the product preorder, as we observed in Remark 5.3.

In an $S$-protomodular category $\mathbf{C}$, it is possible to identify a protomodular subcategory, called the protomodular core relative to the class $S$. Its objects are the so-called $S$-special objects:

Definition 6.3. Let $\mathbf{C}$ be an $S$-protomodular category. An object $X$ is said to be $S$-special when the point

$$
X \stackrel{\langle 1,0\rangle}{\longrightarrow} X \times X \underset{\pi_{2}}{\stackrel{\langle 1,1\rangle}{\leftrightarrows}} X
$$

belongs to the class $S$.

Being OrdGrp an $S$-protomodular category for the class $S$ considered above, we can wonder what is the protomodular core of OrdGrp w.r.t. the class $S$. If the point

$$
X \stackrel{\langle 1,0\rangle}{\longrightarrow} X \times X \underset{\pi_{2}}{\stackrel{\langle 1,1\rangle}{\leftrightarrows}} X
$$

is in $S$, the map $q: X \times X \rightarrow X$ is given by $q(x, y)=x-y$. It is easy to see that $q$ is monotone if and only if the inversion map $i: X \rightarrow X$ sending $x$ to $-x$ is monotone. This happens if and only if $P_{X}$ is a group. Hence:

Proposition 6.4. The protomodular core S-OrdGrp is the full subcategory of OrdGrp whose objects are the preordered groups $X$ for which $P_{X}$ is a group, i.e. those for which the preorder relation is symmetric.

Now we study another approach to $S$-protomodularity, which gives the same protomodular core as the previous one.

Let $\operatorname{Mon}(\mathbf{C})$ be the category of internal monoids in a finitely complete category $\mathbf{C}$. The notion of a Schreier point only involves finite limits, so it is possible to express it internally in C. Moreover, the notion of $S$-protomodularity only involves finite limits and monomorphisms (indeed, two morphisms are jointly strongly epimorphic if, whenever they factor through a monomorphism $m, m$ is an isomorphism), hence it is Yoneda invariant (see Example 2.5 in [11]). This means the following:

Proposition 6.5. $\operatorname{Mon}(\mathbf{C})$ is an $S$-protomodular category w.r.t. the class $S$ of internal Schreier points. 
When $\mathbf{C}=$ Set, the protomodular core is the category Grp of groups. Indeed, if $X$ is a group, the map $q: X \times X \rightarrow X$ defined by $q(x, y)=x-y$ satisfies the Schreier condition. Conversely, suppose that the point

$$
X \stackrel{\langle 1,0\rangle}{\longrightarrow} X \times X \underset{\pi_{2}}{\stackrel{\langle 1,1\rangle}{\longleftrightarrow}} X
$$

is a Schreier one. Then for all $(x, y) \in X \times X$ there exists a unique element $q(x, y) \in X$ such that

$$
(x, y)=(q(x, y), 0)+(y, y) .
$$

From this equality we get that $x=q(x, y)+y$. In particular, choosing $x=0$ we prove that every $y \in X$ is left invertible, hence $X$ is a group.

Now, the argument above can be internalized, giving that the protomodular core of $\mathbf{M o n}(\mathbf{C})$ w.r.t. the class of internal Schreier points is the full subcategory $\operatorname{Grp}(\mathbf{C})$ of internal groups in $\mathbf{C}$. When $\mathbf{C}=\mathbf{T o p}$, we conclude that the protomodular core of the category of topological monoids w.r.t. the class of topological Schreier points is just the category of topological groups. When $\mathbf{C}=$ Ord, the category $\mathbf{M o n}(\mathbf{O r d})$ is just the category of preordered monoids, i.e. those monoids equipped with a preorder relation such that the monoid operation is monotone. The protomodular core of Mon(Ord) w.r.t. the class of preordered Schreier points is the subcategory of internal groups in Ord, which are precisely those preordered groups for which the preorder relation is symmetric, i.e. the positive cone is a group.

We conclude by observing that OrdGrp is a subcategory of Mon(Ord). Moreover, every point in OrdGrp, whose restriction to the positive cones is a Schreier point in Mon, is actually an internal Schreier point in Mon(Ord). This means that the class of points we considered in the second approach contains the class of the first one.

\section{ACKNOWLEDGEMEnTS}

This work was partially supported by Centro de Matemática da Universidade de Coimbra - UID/MAT/00324/2013, funded by the Portuguese Government through FCT/MCTES and co-funded by the European Regional Development Fund through the Partnership Agreement PT2020, and by the FCT Sabbatical Grant SFRH/BSAB/127925/2016.

This work was partially supported by the Fundação para a Ciência e a Tecnologia (FCT) and Centro2020 through the Project references:

UID/Multi/04044/2013 and PAMI - ROTEIRO/0328/2013 (N. 022158).

This work was partially supported by the Programma per Giovani Ricercatori "Rita LeviMontalcini", funded by the Italian government through MIUR.

\section{REFERENCES}

[1] M. Barr, Exact categories, in Lecture Notes in Mathematics, vol. 236 (1971), Springer-Verlag, 1-120.

[2] F. Borceux, D. Bourn, Mal'cev, protomodular, homological and semi-abelian categories, Mathematics and its Applications, vol. 566 (2004), Kluwer.

[3] F. Borceux, M.M. Clementino, Topological semi-abelian algebras, Adv. Math. 190 (2005), 425-453.

[4] F. Borceux, M.M. Clementino, Topological protomodular algebras, Topology Appl. 153 (2006), 3085-3100.

[5] D. Bourn, Normalization equivalence, kernel equivalence and affine categories, in Lecture Notes in Mathematics, vol. 1488 (1991), Springer-Verlag 43-62.

[6] D. Bourn, Mal'cev categories and fibration of pointed objects, Appl. Categ. Structures 4 (1996), 307-327. 
[7] D. Bourn, Baer sums and fibered aspects of Malcev operations, Cah. Topol. Géom. Différ. Catég. 40 (1999), 297-316.

[8] D. Bourn, Z. Janelidze, Pointed protomodularity via natural imaginary subtractions, J. Pure Appl. Algebra 213 (2009), 1835-1851.

[9] D. Bourn, N. Martins-Ferreira, A. Montoli, M. Sobral, Schreier split epimorphisms in monoids and in semirings, Textos de Matemática (Série B), Departamento de Matemática da Universidade de Coimbra, vol. 45 (2013).

[10] D. Bourn, N. Martins-Ferreira, A. Montoli, M. Sobral, Monoids and pointed S-protomodular categories, Homology Homotopy Appl. 18 n.1 (2016), 151-172.

[11] D. Bourn, A. Montoli, The $3 \times 3$ lemma in the $\Sigma$-Mal'tsev and in the $\Sigma$-protomodular settings. Applications to monoids and quandles, submitted, preprint arXiv:1801.09104v1.

[12] P.M. Cohn, Universal algebra, Mathematics and Its Applications 6, D. Reidel Publishing Company, 1981.

[13] Z. Janelidze, The pointed subobject functor, $3 \times 3$ lemmas, and subtractivity of spans, Theory Appl. Categ. 23 (2010), No. 11, 221-242.

[14] J. MacDonald, M. Sobral, Aspects of monads, in M.C. Pedicchio, W. Tholen (eds.), Categorical Foundations, Encyclopedia of Mathematics and its Applications, Cambridge University Press, 2004, 213-268.

[15] A. I. Mal'cev, On the immersion of an algebraic ring into a field, Math. Ann. 113 (1937), 686-691.

[16] A. I. Mal'cev, On the immersion of associative systems into groups, I, Mat. Sbornik N. S. 6 (1939), 331-336.

[17] A. I. Mal'cev, On the immersion of associative systems into groups, II, Mat. Sbornik N. S. 8 (1940), $241-264$.

[18] N. Martins-Ferreira, Weakly Mal'cev categories, Theory Appl. Categ. 21 (2008), No. 6, 91-117.

[19] N. Martins-Ferreira, New wide classes of weakly Mal'tsev categories, Appl. Categ. Structures 23 (2015), No. $5,741-751$.

[20] N. Martins-Ferreira, Normalized Bicategories Internal to Groups and more General Mal'tsev Categories, Appl. Categ. Structures 25 (2017), No. 6, 1137-1158.

[21] N. Martins-Ferreira, A. Montoli, M. Sobral, Semidirect products and crossed modules in monoids with operations, J. Pure Appl. Algebra 217 (2013), 334-347.

[22] A. Montoli, D. Rodelo, T. Van der Linden, Two characterisations of groups amongst monoids, J. Pure Appl. Algebra 222 (2018), 747-777.

CMuC, Department of Mathematics, University of Coimbra, 3001-501 Coimbra, Portugal

E-mail address: mmc@mat.uc.pt

CDRsP, ESTG, Instituto Politécnico de Leiria, Portugal

E-mail address: martins.ferreira@ipleiria.pt

Dipartimento di Matematica "Federigo Enriques", Università degli Studi di Milano, Via Saldini 50, 20133 Milano, Italy

E-mail address: andrea.montoli@unimi.it 